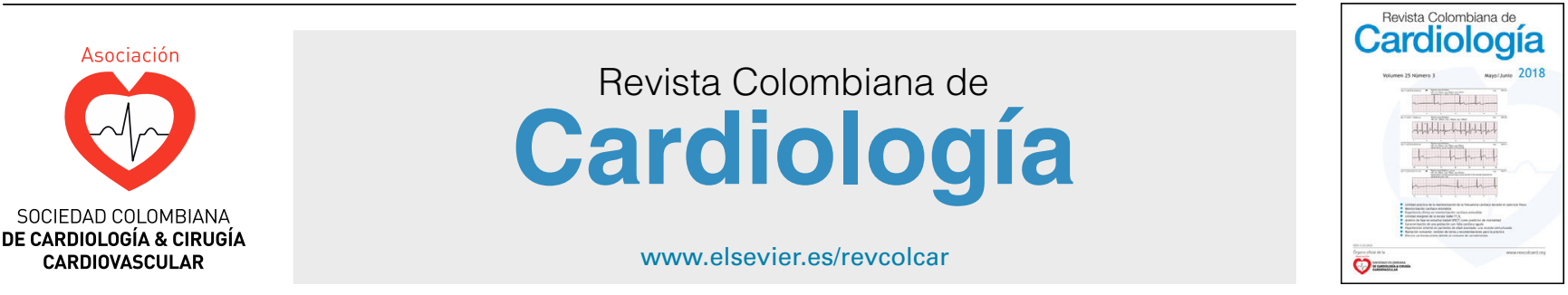

CARDIOLOGÍA DEL ADULTO - ARTÍCULO ORIGINAL

\title{
Desenlaces materno-fetales en embarazadas con categoría de riesgo III - IV según la Organización Mundial de la Salud en un centro de tercer nivel durante 2006 a 2017
}

\author{
Miguel L. Acosta-Calderón ${ }^{a}$, Ana G. Múnera-Echeverri ${ }^{\mathrm{b}, *}$, \\ Rubén D. Manrique-Hernández ${ }^{c}$ y Elsa $M$. Vásquez-Trespalacios ${ }^{d}$ \\ a Centro Clínico y de Investigación SICOR, Clínica Medellín. Medellín, Colombia \\ b Servicio de Cardiología no invasiva, Hospital General de Medellín Luz Castro de Gutiérrez. Medellín, Colombia \\ c Gestión del Conocimiento, Universidad CES. Medellín, Colombia \\ d Universidad CES. Medellín, Colombia
}

Recibido el 17 de septiembre de 2018; aceptado el 1 de diciembre de 2019

Disponible en Internet el 15 de septiembre de 2020

\section{PALABRAS CLAVE \\ Embarazo; \\ Enfermedad cardiovascular; \\ Desenlaces maternos; \\ Desenlaces fetales}

\begin{abstract}
Resumen
Objetivo: describir los desenlaces materno-fetales en embarazadas con categoría de riesgo III-IV según la Organización Mundial de la Salud (OMS).

Métodos: se revisaron las historias clínicas de 41 embarazadas con categoría de riesgo III-IV según la OMS, atendidas en un hospital de tercer nivel en Medellín, Colombia. Se recolectaron variables demográficas, clínicas y ecocardiográficas en un formulario diseñado para tal fin. Las variables primarias están orientadas a la evaluación de los desenlaces maternos y fetales. Los análisis estadísticos se realizaron con el software IBM-SPSS versión 23.

Resultados: la tasa de eventos neonatales fue mayor que la tasa de eventos maternos (68.3 vs. $31.7 \%$ ). Los desenlaces maternos primarios y secundarios ocurrieron en un 31.7 y $12.2 \%$ respectivamente. La distribución según la categoría de riesgo de la OMS fue del $7.3 \%$ en el grupo III y del $\mathbf{9 2 . 7 \%}$ en el grupo IV. Solo hubo una muerte materna no relacionada con enfermedad cardiovascular. La presencia de insuficiencia tricuspídea moderada/severa se asoció con prematuridad ( $\mathrm{p}$ 0.006) y las gestantes con presión sistólica pulmonar $\geq 50 \mathrm{~mm} \mathrm{Hg}$ tuvieron mayor número de neonatos con distrés respiratorio ( $p$ 0.010). La falla cardiaca derecha se relacionó con muerte materna ( $p$ 0.014) y prematuridad ( $p$ 0.019), mientras que la de falla cardiaca izquierda se asoció con muerte neonatal ( $\mathrm{p}$ 0.003).
\end{abstract}

\footnotetext{
* Autor para correspondencia.

Correo electrónico: anagm@une.net.co (A.G. Múnera-Echeverri).
} 
Conclusiones: la enfermedad cardiovascular materna es causa frecuente de alta morbilidad y mortalidad materno-fetal. Este estudio identificó los principales desenlaces maternos y fetales, sin embargo, se requieren estudios con mayor tamaño de muestra.

(c) 2020 Sociedad Colombiana de Cardiología y Cirugía Cardiovascular. Publicado por Elsevier España, S.L.U. Este es un artículo Open Access bajo la licencia CC BY-NC-ND (http:// creativecommons.org/licenses/by-nc-nd/4.0/).

\section{KEYWORDS}

Pregnancy;

Cardiovascular

disease;

Maternal outcomes;

Fetal outcomes

\section{Maternal-fetal outcomes in pregnant women with World Health Organisation risk category III - IV in a tertiary care centre from 2006 to 2017}

\begin{abstract}
Objective: To describe the maternal-fetal outcomes in pregnant woman with risk classification of III-IV according to the World Health Organization (WHO).

Methods: A review was carried out on the medical records of 41 pregnant women with WHO risk classification of III-IV, treated in a tertiary hospital in Medellin, Colombia. Demographic, clinical, and cardiac ultrasound variables were recorded on a form designed for this purpose. The primary variables were directed at evaluating the fetal and maternal outcomes. The statistical analysis was performed using IBM-SPSS software version 23.

Results: The neonatal events rate was greater than the maternal events rate $(68.3 \% \mathrm{vs.} 31.7 \%)$. Primary and secondary outcomes occurred in $31.7 \%$ and $12.2 \%$, respectively. The distribution according to WHO risk category was $7.3 \%$ in risk group III, and $92.7 \%$ in group IV. There was only one maternal death unrelated to cardiovascular disease. The presence of moderate-severe tricuspid insufficiency was associated with prematurity $(P=.006)$, and the mothers with a pulmonary systolic pressure $\geq 50 \mathrm{~mm} \mathrm{Hg}$ had a greater number of newborns with respiratory distress $(P=.010)$. Right heart failure was associated with maternal death $(P=.014)$ and prematurity $(P=.019)$, whilst left heart failure was associated with neonatal death $(P=.003)$.

Conclusions: Maternal cardiovascular disease is a frequent cause of high maternal-fetal morbidity and mortality. Although this study identified the main maternal and fetal outcomes, studies with a greater sample size are required.

(c) 2020 Sociedad Colombiana de Cardiología y Cirugía Cardiovascular. Published by Elsevier España, S.L.U. This is an open access article under the CC BY-NC-ND license (http:// creativecommons.org/licenses/by-nc-nd/4.0/).
\end{abstract}

\section{Introducción}

El número de gestantes en riesgo de sufrir complicaciones cardiovasculares ha aumentado progresivamente, razón que debe motivar la búsqueda temprana de factores de riesgo cardiovascular mayor para evitar complicaciones y mejorar los desenlaces tanto para la madre como para el feto ${ }^{1-3}$.

En la actualidad no se comprende cuál es el impacto del embarazo en la progresión de la enfermedad cardiovascular o cómo la enfermedad cardiovascular incide en los desenlaces del embarazo. El espectro completo de enfermedad cardíaca estructural incluye enfermedad cardíaca congénita, enfermedad valvular cardíaca, enfermedad cardíaca isquémica y miocardiopatías ${ }^{4}$.

Entre el 0.2 al 4\% de los embarazos se complican por enfermedades cardiovasculares, y son estas últimas la principal causa de muerte durante la gestación, con un 10 a un $15 \%^{5-8}$. En Occidente la cardiopatía congénita es la enfermedad cardiovascular más frecuente durante el embarazo (75-82\%); sin embargo, los grandes avances en ayudas diagnósticas, así como las estrategias de manejo médico y quirúrgico han mejorado la sobrevida a largo plazo de las gestantes con cardiopatías congénitas y enfermedad cardiovascular en general, pudiéndose llevar la gestación a feliz término en muchos $\operatorname{casos}^{2,3,9-11}$. En un estudio publicado por nuestro grupo de investigación, sobre enfermedad cardíaca y embarazo, se encontró que, en una región de Colombia, la cardiopatía congénita es la enfermedad cardiovascular más frecuente durante el embarazo ${ }^{12}$.

El riesgo de desenlaces obstétricos y neonatales debe ser evaluado por cuanto es clara la relación entre cardiopatía y embarazo ${ }^{13}$. Los desenlaces adversos relacionados con el feto también son mucho más frecuentes en gestantes con enfermedad cardiovascular, especialmente en aquellas embarazadas que padecen cardiopatías congénitas complejas. Se han reportado tasas de parto prematuro muy altas (16\%), ruptura prematura de membranas y hemorragia posparto (32\%), complicaciones neonatales entre las que se destacan el síndrome de distrés respiratorio y la hemorragia intraventricular (20-28\%), recién nacidos pequeños para la edad gestacional, mortalidad fetal del 4\%, abortos espontáneos (15-25\%) y abortos electivos $^{11,14,15}$.

Para evaluar el riesgo del embarazo se deben tener en cuenta predictores de riesgo generales, como la clase funcional de la Asociación del Corazón de Nueva York (conocida por su sigla en inglés NYHA, por New York Heart Association) y la fracción de eyección del ventrículo izquierdo (FEVI), y 
específicos, como la clasificación modificada de la OMS del riesgo cardiovascular materno ${ }^{2}$.

Hoy se recomienda evaluar el riesgo materno según la clasificación del riesgo modificada de la OMS, pues esta clasificación integra los factores de riesgo cardiovasculares conocidos, incluida la cardiopatía de base y cualquier otra comorbilidad $^{16}$. Así, por ejemplo, las pacientes con enfermedades del grupo III según la clasificación de la OMS tienen un riesgo elevado de morbilidad y mortalidad materna, de tal manera que se requiere la orientación de un especialista para definir si es o no prudente la concepción, o si ya se encuentra embarazada, para determinar si se continúa con el embarazo, en cuyo caso se requerirá monitorización estricta durante toda la gestación, el parto y el puerperio la cual deberá estar a cargo de un grupo multidisciplinario con alta experiencia en el manejo de este tipo de pacientes. Por otro lado, las pacientes con enfermedades del grupo IV, según la OMS, tienen un riesgo extremadamente alto de morbilidad y mortalidad materna, lo que determina que en este escenario el embarazo esté contraindicado. En caso de que este último ocurra se debe valorar y decidir su interrupción; si se decide continuarlo se deberá tratar con las mismas consideraciones mencionadas para el grupo $\mathrm{III}^{2,3,9}$.

En nuestro medio muchas de las pacientes embarazadas que requieren valoración cardiovascular por tener una condición clínica del grupo III-IV según la OMS, ya se encuentran en una etapa avanzada de la gestación, generalmente en el tercer trimestre, etapa en la que la mayoría de los cambios hemodinámicos se han establecido. Sin embargo, dependerá del adecuado manejo y seguimiento multidisciplinario, que la gestación tenga desenlaces favorables tanto para la madre como para el feto. Ese, precisamente, es el objetivo de este estudio: identificar cuáles fueron los desenlaces maternos y fetales en este grupo de pacientes de alto y muy alto riesgo atendidas en un hospital de tercer nivel en la ciudad de Medellín, Colombia.

\section{Materiales y métodos}

Se realizó un estudio descriptivo, con intención analítica, basado en la revisión retrospectiva de las historias clínicas de 41 pacientes embarazadas con categoría de riesgo III y IV según la clasificación modificada de la OMS, atendidas en El Hospital General de Medellín, Colombia, en el periodo comprendido entre enero de 2006 a diciembre de 2017. Se recolectaron datos de variables demográficas, clínicas y los resultados de los estudios ecocardiográficos en un formulario diseñado para tal fin. El Hospital General de Medellín es un centro de referencia para enfermedad cardiovascular y gineco-obstetricia. Cuenta con un grupo multidisciplinario que incluye cardiología clínica, cardiología no invasiva, cardiología intervencionista (hemodinámica y electrofisiología), gineco-obstetricia, neonatología, anestesia general y cardiovascular, cuidado intensivo adulto y neonatal, cardiología pediátrica y cirugía cardiovascular.

Se utilizó estadística descriptiva; las variables continuas se expresaron en forma de promedios y desviaciones estándar, o en medianas y rangos intercuartílicos según la distribución de la variable, en tanto que las variables categóricas se expresaron como proporciones.
Se crearon dos grupos de pacientes teniendo en cuenta la clasificación de riesgo modificada de la OMS. En un grupo se clasificó a las pacientes en categoría de riesgo III en el que se incluyeron: válvula mecánica, ventrículo derecho sistémico, circulación de Fontan, cardiopatía cianótica no reparada, otras cardiopatías congénitas complejas, dilatación aórtica de 40-45 mm en síndrome de Marfan, dilatación aórtica de 45-50 mm en enfermedad aórtica asociada con válvula aórtica bicúspide. En otro grupo se clasificó a las pacientes en categoría de riesgo IV, en el que se incluyeron: hipertensión pulmonar de cualquier causa, disfunción ventricular izquierda severa (FEVI <30\% / NYHA III-IV), cardiopatía periparto previa con cualquier alteración residual de la FEVI, estenosis mitral severa y estenosis valvular aórtica severa sintomática, dilatación aórtica mayor a $45 \mathrm{~mm}$ en síndrome de Marfan, dilatación aórtica mayor a $50 \mathrm{~mm}$ en enfermedad aórtica asociada con válvula aórtica bicúspide y coartación aórtica nativa severa.

Para el diagnóstico de hipertensión pulmonar se tuvo en cuenta el valor de presión sistólica pulmonar (PSP) obtenido mediante estudio ecocardiográfico y cuyo valor fuera mayor o igual a $50 \mathrm{~mm} \mathrm{Hg}$.

Se realizó una comparación para cada una de las variables descriptivas definidas. Para la comparación de las variables categóricas se utilizó una prueba exacta de Fisher según la frecuencia encontrada. Para todas las comparaciones se consideró un valor $\mathrm{p}<0.05$ como estadísticamente significativo. Todos los análisis se realizaron con el software IBM-SPSS versión 23 .

\section{Resultados}

La población estuvo conformada por 41 pacientes embarazadas con enfermedad cardiovascular que se encontraban en categoría de riesgo III-IV según la clasificación modificada de la Organización Mundial de la Salud (OMS). La distribución de las pacientes, según la categoría de riesgo de la OMS, fue del $7.3 \%$ en el grupo III y del $92.7 \%$ en el grupo IV. La tabla 1 describe las características de la población estudiada.

\section{Características clínicas y sociodemográficas}

El promedio de edad fue de 25 años (rango 16-44). Al momento del estudio ecocardiográfico la edad gestacional promedio fue de 28.7 semanas (rango 8-40), dato que deja claro que la mayoría de las pacientes consultaron en etapas avanzadas de su gestación. Solo el $36.6 \%$ tenía estudios de bachillerato completo, lo cual refleja el bajo nivel académico de nuestra población de estudio. Llama la atención que el $43.9 \%$ de las pacientes habían estado previamente embarazadas, lo que supone que ya habían tenido evaluación por especialista en Obstetricia; sin embargo, no habían recibido la asesoría necesaria respecto al riesgo y contraindicación del embarazo, o ambas, por encontrarse en categoría III-IV según la OMS. Pese a que las pacientes estaban en categoría de riesgo III-IV de la OMS, la mayoría tenían clase funcional NYHA I antes del embarazo (63.4\%), el 36.6\% restante eran clase funcional NYHA II y ninguna se encontraba en clase funcional III-IV de la NYHA. Durante el embarazo la clase funcional según la NYHA era III-IV en el 29.3\%, probablemente por el impacto hemodinámico del embarazo sumado a la 
Tabla 1 Características clínicas y sociodemográficas de embarazadas con categoría de riesgo OMS III-IV atendidas en el Hospital General de Medellín, 2006-2017

\begin{tabular}{|c|c|}
\hline & $\begin{array}{l}\text { Número de } \\
\text { pacientes } \\
n=41\end{array}$ \\
\hline Edad (años), promedio $\pm D E$ & $25.1 \pm 8.1$ \\
\hline $\begin{array}{l}\text { Edad gestacional durante la } \\
\text { ecocardiografía (semanas), promedio } \\
\pm D E\end{array}$ & $28,7 \pm 8.4$ \\
\hline \multicolumn{2}{|l|}{ Etnia, $n(\%)$} \\
\hline Blanca & $0(0.0)$ \\
\hline Negra & $2(4.9)$ \\
\hline Indígena & $1(2.4)$ \\
\hline Mestiza & $38(92.7)$ \\
\hline \multicolumn{2}{|l|}{ Nivel educativo, $n$ (\%) } \\
\hline Primaria incompleta & $9(22)$ \\
\hline Primaria completa & $12(29.3)$ \\
\hline Bachiller incompleto & $5(12.2)$ \\
\hline Bachiller completo & $15(36.6)$ \\
\hline Técnico / Universitario & $0(0.0)$ \\
\hline \multicolumn{2}{|l|}{ NYHA antes del embarazo, $n(\%)$} \\
\hline NYHA clase I & $26(63.4)$ \\
\hline NYHA clase II & $15(36.6)$ \\
\hline NYHA clase III & $0(0.0)$ \\
\hline NYHA clase IV & $0(0.0)$ \\
\hline \multicolumn{2}{|l|}{ NYHA durante el embarazo, $n$ (\%) } \\
\hline NYHA clase I & $11(26.8)$ \\
\hline NYHA clase II & $18(43.9)$ \\
\hline NYHA clase III & $10(24.4)$ \\
\hline NYHA clase IV & $2(4.9)$ \\
\hline \multicolumn{2}{|l|}{ Historia obstétrica, n (\%) } \\
\hline Primigestante & $23(56)$ \\
\hline Secundigestante & $9(22)$ \\
\hline Multigestante ( $\geq 3$ embarazos) & $9(22)$ \\
\hline \multicolumn{2}{|l|}{ Antecedentes personales, $n(\%)$} \\
\hline Hipertensión arterial & $9(22)$ \\
\hline Tabaquismo & $3(7.3)$ \\
\hline Falla cardíaca & $4(9.8)$ \\
\hline Diabetes mellitus tipo 2 & $1(2.4)$ \\
\hline \multicolumn{2}{|c|}{ Medicación cardiovascular durante la gestación, n (\%) } \\
\hline ASA & $3(7.3)$ \\
\hline Warfarina & $4(9.8)$ \\
\hline Betabloqueadores & $14(34.1)$ \\
\hline Heparinas & $18(43.9)$ \\
\hline Diuréticos & $13(31.7)$ \\
\hline Calcioantagonistas & $5(12.2)$ \\
\hline
\end{tabular}

ASA: ácido acetil salicílico, DE: desviación estándar; NYHA: New York Heart Association.

presencia de enfermedad cardiovascular. Durante el embarazo las pacientes con clase funcional IV de la NYHA fueron las que más tuvieron eventos cardíacos maternos primarios, ataque cerebrovascular (ACV) / isquemia cerebral transitoria (ICT), insuficiencia cardíaca congestiva (ICC) / edema pulmonar ( $p$ 0.001) y deterioro de la clase funcional mayor a dos clases.

En la tabla 2 se describen los hallazgos ecocardiográficos relevantes y los diagnósticos según la categoría de riesgo de la OMS.
Tabla 2 Hallazgos ecocardiográficos

\begin{tabular}{ll}
\hline Hallazgo ecocardiográfico & $\begin{array}{l}\text { Número de } \\
\text { pacientes } \\
\mathrm{n}=41\end{array}$ \\
\hline FEVI (\%), n (\%) & $2(4.9)$ \\
$<30$ & $2(4.9)$ \\
$30-40$ & $8(19.5)$ \\
$41-55$ & $29(70.7)$ \\
$>55$ & \\
PSP (mm Hg), n (\%) & $11(26.8)$ \\
$<50$ & $23(56.1)$ \\
$50-70$ & $7(17.1)$ \\
$>70$ & \\
Volumen indexado de Al (ml/m²), n (\%) & $21(51.2)$ \\
$<35$ & $6(14.6)$ \\
$35-41$ & $2(4.9)$ \\
$42-48$ & $12(29.3)$ \\
$>48$ & $14(34.1)$ \\
Insuficiencia tricuspídea moderada/severa, & \\
$\mathrm{n}$ (\%) & $10(24.4)$ \\
Disfunción del VD, $\mathrm{n}(\%)$ & \\
Categoría OMS, $\mathrm{n}(\%)$ & $3(7.3)$ \\
OMS III & $38(92.7)$ \\
OMS IV &
\end{tabular}

Al: aurícula izquierda; FEVI: fracción de eyección del ventrículo izquierdo; OMS: Organización Mundial de la Salud; PSP: presión sistólica pulmonar; VD: ventrículo derecho.

En cuanto a los hallazgos ecocardiográficos, la mayoría de pacientes tenían fracción de eyección del ventrículo izquierdo (FEVI) normal (70.7\%), disfunción sistólica leve (19.5\%), disfunción sistólica moderada (4.9\%) y disfunción sistólica severa (4.9\%). Las pacientes que tenían FEVI mayor al 55\% tuvieron mayor número de recién nacidos vivos. En cuanto a la presión sistólica pulmonar (PSP), el $17.1 \%$ tenía valores mayores a $70 \mathrm{~mm} \mathrm{Hg}$, el $56,1 \%$ entre $50-70 \mathrm{~mm}$ $\mathrm{Hg}$ y el $26.8 \%$ valores menores a $50 \mathrm{~mm} \mathrm{Hg}$. Los recién nacidos de madres con PSP mayor o igual a $50 \mathrm{~mm}$ Hg tuvieron mayor frecuencia de distrés respiratorio. En cuanto al volumen indexado de la aurícula izquierda, el 51.2\% tenía volúmenes normales, el $29.3 \%$ crecimiento severo de la aurícula izquierda, el $4.9 \%$ crecimiento moderado y el $14.6 \%$ crecimiento leve. El 34.1\% tenía insuficiencia tricuspídea moderada o severa, hallazgo que se relacionó con prematuridad.

\section{Desenlaces maternos}

Los desenlaces maternos son descritos en la tabla 3. En la población total del estudio, la vía del parto preferida fue por cesárea en el $63.4 \%$ y la indicación de esta obedeció a causas maternas en el $38.4 \%$, causas fetales en el $34.6 \%$ y causas cardíacas en el $26.9 \%$. Requirieron manejo en unidad de cuidados intensivos ( $\mathrm{UCl}$ ) el $82.9 \%$ y necesidad de medicación cardiovascular endovenosa el $26.8 \%$.

El antecedente de tabaquismo se asoció con el desarrollo de hemorragia postparto y el de falla cardíaca se asoció con el desarrollo durante el embarazo de ICC/Edema pulmonar. La presencia de falla cardíaca derecha se asoció con 
Tabla 3 Desenlaces maternos

\begin{tabular}{ll}
\hline & $\begin{array}{l}\text { Número de } \\
\text { pacientes } \\
\mathrm{n}=41\end{array}$ \\
\hline $\begin{array}{l}\text { Vía del parto, } \mathrm{n}(\%) \\
\text { Cesárea }\end{array}$ & $26(63.4)$ \\
Vaginal & $8(19.5)$ \\
Vaginal + fórceps & $7(17.1)$ \\
Indicación de la cesárea, $\mathrm{n}(\%)$ & \\
Materna & $10(38.4)$ \\
Fetal & $9(34.6)$ \\
Cardíaca & $7(26.9)$ \\
Necesidad de manejo en UCI, $\mathrm{n}(\%)$ & $34(82.9)$ \\
Hemorragia postparto, $\mathrm{n}(\%)$ & $1(2.4)$ \\
Necesidad de medicación & $11(26.8)$ \\
cardiovascular IV, $\mathrm{n}(\%)$ & \\
Tipo de anestesia, $\mathrm{n}(\%)$ & \\
General & $4(9.8)$ \\
Conductiva & $35(85.4)$ \\
No anestesia & $2(4.9)$ \\
Desarrollo de THAE, $\mathrm{n}(\%)$ & $12(29.3)$ \\
Desarrollo de RPM, $\mathrm{n}(\%)$ & $3(7.3)$ \\
\hline V:
\end{tabular}

IV: intravenoso; RPM: ruptura prematura de membranas; THAE: trastorno hipertensivo asociado al embarazo; UCl: unidad de cuidados intensivos.

muerte materna, mientras que la presencia de falla cardíaca izquierda durante el embarazo se relacionó con la necesidad de medicación cardiovascular intravenosa, ruptura prematura de membranas, desarrollo de edema pulmonar y ACV/TIA. El desarrollo de arritmia sintomática sostenida que requirió tratamiento se asoció con el antecedente de tabaquismo, la presencia de insuficiencia tricuspídea moderada/severa, uso de warfarina durante el embarazo y crecimiento de la aurícula izquierda moderado/severo (tabla 4).

\section{Eventos maternos primarios y secundarios}

Respecto a los eventos maternos primarios, el $17.1 \%$ desarrolló ICC/edema pulmonar, principalmente las que tenían FEVI < 55\% (p 0.006), el 2.4\% arritmia sintomática sostenida con necesidad de tratamiento, el $2.4 \%$ ACV/ICT, el $12.2 \%$ necesidad de intervención o procedimiento durante la gestación. Solo hubo una muerte materna, cuya causa obedeció a tromboembolia pulmonar en paciente con antecedente de esplenectomía por púrpura trombocitopénica inmune (PTI). En cuanto a los eventos cardíacos secundarios el $2.4 \%$ tuvo deterioro de la clase funcional mayor a 2 clases de la NYHA; además, el $9.8 \%$ requirió terapia invasiva urgente antes de 6 meses posparto, en especial aquellas que tenían crecimiento de la aurícula izquierda moderado/severo y las que habían desarrollado trastorno hipertensivo asociado al embarazo (THAE). El 2.4\% desarrolló arritmia sintomática no sostenida con necesidad de manejo. Los detalles de eventos maternos primarios y secundarios se describen en las tablas 5 y 6 , respectivamente.

\section{Desenlaces fetales}

En lo concerniente a los desenlaces fetales, el porcentaje de nacidos vivos fue del $90,2 \%$. Por su parte, el $31.7 \%$ fueron neonatos pequeños para la edad gestacional y $56.1 \%$ fueron prematuros. Se registró distrés respiratorio en el $26.8 \%$, hemorragia intracerebral en el $7.3 \%$, abortos $2.4 \%$, muertes neonatales el $7.3 \%$ en el que se incluía un embarazo gemelar y necesidad de manejo en $\mathrm{UCI}$ neonatal en el $34.1 \%$ (tabla 7). El desarrollo de distrés respiratorio fue más frecuente en pacientes con PSP mayor a $50 \mathrm{~mm} \mathrm{Hg}$ (p 0.01). La prematuridad se relacionó con la presencia de insuficiencia tricuspídea moderada/severa ( $p$ 0.006) y falla cardiaca derecha ( $p$ 0.019) en la madre. La muerte neonatal fue más frecuente en los hijos de madres con falla cardiaca izquierda ( $p$ 0.003) y que recibieron warfarina durante la gestación ( $p$ 0.049) (tabla 8).

\section{Predictores de eventos neonatales}

Se encontró obstrucción del ventrículo izquierdo en el $12.2 \%$, clase NYHA III-IV/cianosis en el 31.7\%, embarazo múltiple en el $2.4 \%$, consumo de warfarina durante el embarazo en el $9.8 \%$, falla cardíaca derecha en el $14.6 \%$ y falla cardíaca izquierda en el $19.5 \%$ (tabla 8).

En total se hallaron desenlaces neonatales en el $68.3 \%$ $(n=28)$ y desenlaces maternos en el $31.7 \%(n=13)$. Los diagnósticos encontrados en las categorías de riesgo III y IV según la clasificación de la OMS se describen en la figura 1. El diagnóstico más frecuente fue hipertensión pulmonar de cualquier etiología en el $61 \%(n=25)$; entre la clasificación de hipertensión pulmonar el $76 \%$ correspondía a la categoría 1 y el $24 \%$ a la categoría 2 . Entre la categoría 1 de hipertensión pulmonar, el $5.2 \%$ correspondía a hipertensión arterial primaria y el $94.8 \%$ a las asociadas a enfermedad cardíaca congénita (tabla 9).

\section{Discusión}

En este estudio la tasa de eventos neonatales fue considerablemente mayor a la tasa de eventos maternos $(68.3$ vs. $31.7 \%$ ). Respecto a los desenlaces maternos primarios y secundarios, estos ocurrieron en un 31.7 y $12.2 \%$ respectivamente.

La enfermedad cardiovascular materna es una causa frecuente de alta morbilidad y mortalidad tanto para la madre como para el feto ${ }^{17}$. En Estados Unidos la enfermedad cardiovascular es la primera causa de muerte materna relacionada con el embarazo y la mortalidad fetal total entre los nacidos de madres con enfermedad cardíaca alcanza el $1.7 \%^{7}$. En este estudio la mortalidad materna fue del $2.4 \%$ y la neonatal fue del $7.2 \%$.

Los desenlaces neonatales adversos, como muertes neonatales y fetales, prematuridad, pequeño para la edad gestacional, distrés respiratorio y hemorragia intraventricular, fueron más frecuentes en embarazadas con enfermedad cardiovascular $^{18-20}$. En el estudio de Khairy et al. ${ }^{21}$ el porcentaje de desenlaces neonatales adversos fue del $28 \%$, incluyendo prematuridad (21\%), pequeño para la edad gestacional (8\%), muerte fetal intrauterina (3\%), hemorragia intraventricular (1.4\%) y muerte neonatal $(1.4 \%)$. Los 


\begin{tabular}{|c|c|c|c|c|c|c|c|c|c|c|c|c|}
\hline \multirow[t]{2}{*}{ Variable } & \multicolumn{2}{|c|}{ ICC-Edema pulmonar } & \multirow[t]{2}{*}{ Valor de $p$} & \multicolumn{2}{|c|}{$\begin{array}{l}\text { Ruptura prematura de } \\
\text { membranas }\end{array}$} & \multirow[t]{2}{*}{ Valor de $p$} & \multicolumn{2}{|l|}{ ACV-TIA } & \multirow[t]{2}{*}{ Valor de $p$} & \multicolumn{2}{|c|}{$\begin{array}{l}\text { Deterioro de la clase } \\
\text { funcional }\end{array}$} & \multirow[t]{2}{*}{ Valor de $\mathrm{p}$} \\
\hline & $\mathrm{Si}$ & No & & $\mathrm{Si}$ & No & & $\mathrm{Si}$ & No & & $\mathrm{Si}$ & No & \\
\hline \multicolumn{13}{|l|}{ FEVI } \\
\hline 0 & $1(14,3 \%)$ & $1(2,9 \%)$ & 0,006 & $0(0 \%)$ & $2(5,3 \%)$ & 0,72 & $0(0 \%)$ & $2(5 \%)$ & 0,935 & $0(0 \%)$ & $2(5 \%)$ & 0,935 \\
\hline 1 & $2(28,6 \%)$ & $0(0 \%)$ & & $0(0 \%)$ & $2(5,3 \%)$ & & $0(0 \%)$ & $2(5 \%)$ & & $0(0 \%)$ & $2(5 \%)$ & \\
\hline 2 & $1(14,3 \%)$ & $7(20,6 \%)$ & & $0(0 \%)$ & $8(21,1 \%)$ & & $0(0 \%)$ & $8(20 \%)$ & & $0(0 \%)$ & $8(20 \%)$ & \\
\hline 3 & $3(42,9 \%)$ & $26(76,5 \%)$ & & $3(100 \%)$ & $26(68,4 \%)$ & & $1(100 \%)$ & $28(70 \%)$ & & $1(100 \%)$ & $28(70 \%)$ & \\
\hline \multicolumn{13}{|c|}{$\begin{array}{l}\text { NYHA antes del } \\
\text { embarazo }\end{array}$} \\
\hline I & $4(57,1 \%)$ & $22(64,7 \%)$ & 0,51 & $0(0 \%)$ & $26(68,4 \%)$ & 0,043 & $0(0 \%)$ & $26(65 \%)$ & 0,366 & $1(100 \%)$ & $25(62,5 \%)$ & 0,634 \\
\hline II & $12(35,3 \%)$ & $2(42,9 \%)$ & & $3(100 \%)$ & $12(31,6 \%)$ & & $1(1 \%)$ & $14(35 \%)$ & & $0(0 \%)$ & $15(37,5)$ & \\
\hline \multicolumn{13}{|c|}{$\begin{array}{l}\text { NYHA durante el } \\
\text { embarazo }\end{array}$} \\
\hline I & $1(14,3 \%)$ & $10(29,4 \%)$ & $<0,001$ & $0(0 \%)$ & $11(28,9 \%)$ & 0,092 & $0(0 \%)$ & $11(27,5 \%)$ & $<0,0001$ & $0(0 \%)$ & $11(27,5 \%)$ & $<0,001$ \\
\hline II & $0(0 \%)$ & $18(52,9 \%)$ & & $1(33,3 \%)$ & $17(44,7 \%)$ & & $0(0 \%)$ & $18(45 \%)$ & & $0(0 \%)$ & $18(45 \%)$ & \\
\hline III & $4(57,1 \%)$ & $6(17,6 \%)$ & & $1(33,3 \%)$ & $9(23,7 \%)$ & & $0(0 \%)$ & $10(25 \%)$ & & $0(0 \%)$ & $10(25 \%)$ & \\
\hline IV & $2(28,6 \%)$ & $0(0 \%)$ & & $1(33,3 \%)$ & $1(2,6 \%)$ & & $1(100 \%)$ & $1(2,5 \%)$ & & $1(100 \%)$ & $1(2,5 \%)$ & \\
\hline
\end{tabular}


Tabla 5 Eventos maternos primarios

\begin{tabular}{|c|c|}
\hline & $\begin{array}{l}\text { Número de } \\
\text { pacientes } \\
n=41\end{array}$ \\
\hline $\begin{array}{l}\text { Desarrollo de ICC/Edema pulmonar, } n \\
\text { (\%) }\end{array}$ & $7(17.1)$ \\
\hline $\begin{array}{l}\text { Arritmia sintomática sostenida que } \\
\text { requiere tratamiento, } \mathrm{n}(\%)\end{array}$ & $1(2.4)$ \\
\hline $\mathrm{ACV} / \mathrm{ICT}, \mathrm{n}(\%)$ & $1(2.4)$ \\
\hline Muerte materna, n (\%) & $1(2.4)$ \\
\hline $\begin{array}{l}\text { Necesidad de intervención o } \\
\text { procedimiento, } \mathrm{n}(\%)\end{array}$ & $5(12.2)$ \\
\hline
\end{tabular}

Tabla 6 Eventos maternos secundarios

\begin{tabular}{ll}
\hline & $\begin{array}{l}\text { Número de } \\
\text { pacientes } \\
\mathrm{n}=41\end{array}$ \\
\hline $\begin{array}{l}\text { Cambios en la NYHA > de } 2 \text { clases, } \mathrm{n} \\
(\%)\end{array}$ & $1(2.4)$ \\
$\begin{array}{l}\text { Recibe terapia invasiva urgente }<6 \\
\text { meses postparto, } \mathrm{n}(\%)\end{array}$ & $4(9.8)$ \\
$\begin{array}{l}\text { Arritmia sintomática no sostenida } \\
\text { que requiere tratamiento, } \mathrm{n}(\%)\end{array}$ & $1(2.4)$ \\
$\begin{array}{l}\text { Ningún evento secundario, } \mathrm{n}(\%) \\
\text { NYHA: New York Heart Association. } \\
\text { *Pueden existir pacientes con más de un evento secundario. }\end{array}$ & $36(87.8)$ \\
\hline
\end{tabular}

Tabla 7 Desenlaces fetales

\begin{tabular}{ll}
\hline & $\begin{array}{l}\text { Número de } \\
\text { pacientes } \\
\mathrm{n}=41\end{array}$ \\
\hline $\begin{array}{l}\text { Pequeño para la edad gestacional, } \mathrm{n} \\
(\%)\end{array}$ & $13(31.7)$ \\
$\begin{array}{l}\text { Prematuridad (<37 semanas), } \mathrm{n}(\%) \\
\text { Distrés respiratorio, } \mathrm{n}(\%)\end{array}$ & $23(56.1)$ \\
Hemorragia intracerebral, $\mathrm{n}(\%)$ & $11(26.8)$ \\
Resultado del producto, $\mathrm{n}(\%)$ & $3(7.3)$ \\
$\begin{array}{l}\text { Aborto } \\
\text { Muerte fetal }\end{array}$ & $1(2.4)$ \\
$\begin{array}{l}\text { Muerte neonatal } \\
\text { Vivo }\end{array}$ & $0(0)$ \\
Necesidad de manejo en UCl & $3(7.3)$ \\
neonatal, $\mathrm{n}$ (\%) & $37(90.2)$ \\
Ningún desenlace fetal, $\mathrm{n}(\%)$ & $14(34.1)$ \\
\hline
\end{tabular}

UCI: Unidad de cuidados intensivos.

resultados en registros previos, incluidos el registro danés de Sillesen et al., el registro australiano y el registro europeo ROPAC, los desenlaces adversos fueron abortos espontáneos, hemorragia postparto, partos prematuros y niños pequeños para la edad gestacional ${ }^{22-24}$.

La prematuridad y el bajo peso al nacer han estado claramente relacionados con la categoría IV de la $\mathrm{OMS}^{7}$. En otro estudio se encontró que, en presencia de enfermedad
Tabla 8 Predictores de eventos neonatales

\begin{tabular}{ll}
\hline & $\begin{array}{l}\text { Número de } \\
\text { pacientes } \\
\mathrm{n}=41\end{array}$ \\
\hline $\begin{array}{l}\text { Obstrucción del VI (gradiente } \\
\text { subaórtico > 30 mm Hg), } \mathrm{n}(\%)\end{array}$ & $5(12.2)$ \\
NYHA III-IV/Cianosis, $\mathrm{n}(\%)$ & $13(31.7)$ \\
Tabaquismo, n (\%) & $3(7.3)$ \\
Embarazo múltiple, n (\%) & $1(2.4)$ \\
Warfarina durante el embarazo, n (\%) & $4(9.8)$ \\
Prótesis valvular mecánica, n (\%) & $3(7.3)$ \\
Arritmia sintomática, $\mathrm{n}(\%)$ & $2(4.9)$ \\
Falla cardíaca derecha, $\mathrm{n}(\%)$ & $6(14.6)$ \\
Falla cardíaca izquierda, $\mathrm{n}(\%)$ & $8(19.5)$ \\
\hline
\end{tabular}

NYHA: New York Heart Association; VI: ventrículo izquierdo. FEVI: fracción de eyección del ventrículo izquierdo; HTP: hipertensión pulmonar; VI: ventrículo izquierdo.

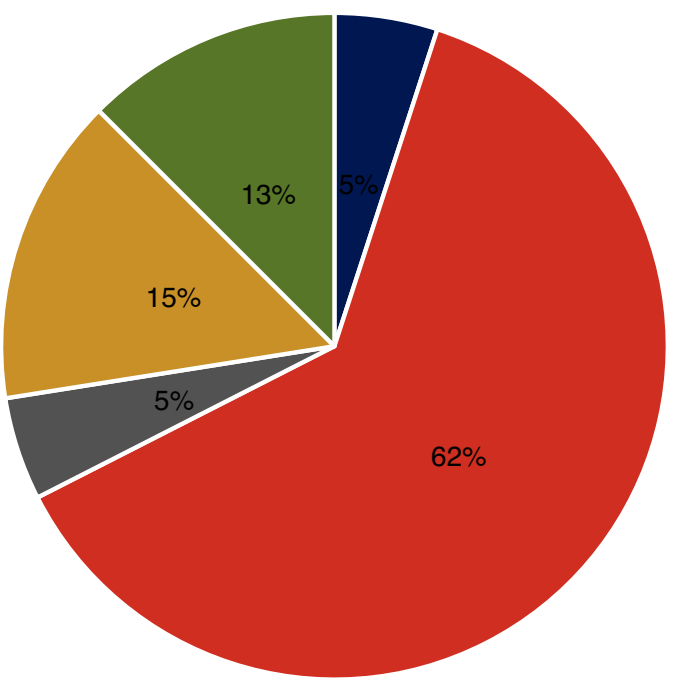

- Prótesis valvular mecánica

- HTP de cualquier etiología

- Disfunción severa del VI (FEVI<30\%)

- Estenosis mitral/aórtica severa sintomática

- Coartación aórtica severa

Figura 1

cardíaca materna, la tasa de partos prematuros es alrededor del $16 \%{ }^{25}$. Con base en los resultados del estudio CARPREG hubo una tasa de complicaciones neonatales del 20\%, entre las que se incluían prematuridad con un $20 \%$, pequeño para la edad gestacional con $4 \%$, muerte fetal con $1 \%$ y muerte neonatal con $1 \% 26$.

Se destaca el alto número de desenlaces adversos neonatales en este estudio donde se incluyen neonatos pequeños para la edad gestacional (31.7\%), prematuridad (56.1\%), distrés respiratorio $(26.8 \%)$, hemorragia intraventricular (7.3\%), necesidad de manejo en UCI neonatal (34.1\%), abortos espontáneos (2.4\%) y muerte neonatal (7.3\%). Estas cifras son significativamente superiores a las reportadas 


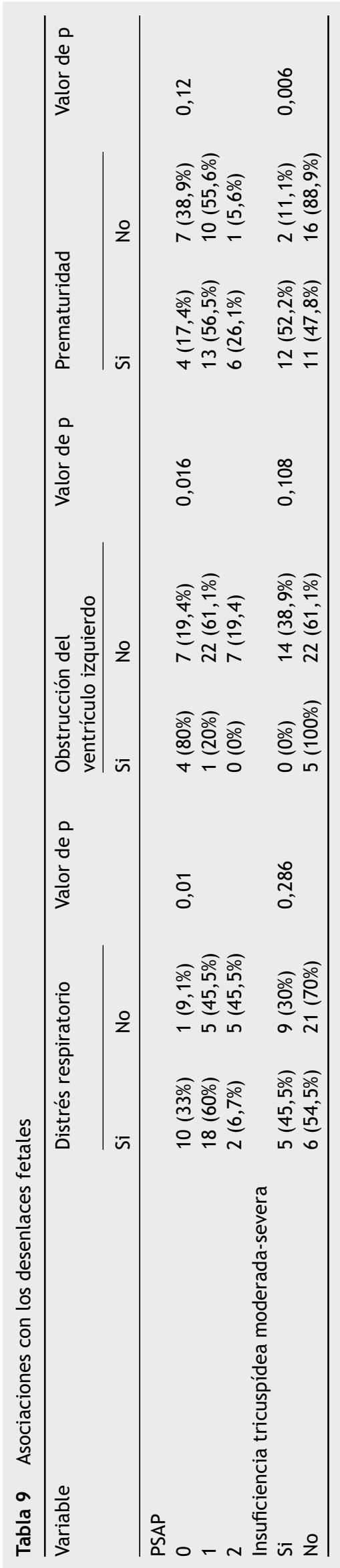

en estudios previos. Sin embargo, a pesar de la tasa tan alta de desenlaces adversos el número de nacidos vivos fue del $90.2 \%$, no hubo muertes fetales y solo se registró una muerte materna no relacionada con enfermedad cardiovascular durante el embarazo.

Es importante resaltar que la mayoría de las pacientes de esta población de estudio tenían su primer contacto con el cardiólogo en etapas tardías de la gestación y preocupante aun, se trataba de las que ya habían estado previamente embarazadas (43.9\%), de quienes se asume debieron contar con asesoría adecuada y acompañamiento de un equipo multidisciplinario. La mayoría de las gestantes se agruparon en categoría de riesgo IV de la OMS (92.7\%) pese a que se encontraban con buena clase funcional antes del embarazo (NYHA I-II). Esto podría explicar, en parte, el porqué eran remitidas de manera tardía al cardiólogo. Otro hallazgo para resaltar es el bajo nivel académico de la población de estudio, pues el $63.4 \%$ de las pacientes no habían culminado sus estudios de educación secundaria, lo que las ubica en una situación de vulnerabilidad ante la toma de decisiones y posiblemente sea una barrera para las consultas oportunas a centros hospitalarios de alta complejidad.

En estudios previos se ha encontrado que el antecedente de falla cardiaca, tabaquismo y el uso de warfarina durante la gestación se asocia con desenlaces adversos maternos y fetales. El grupo de estudio BACH (Boston Adult Congenital Heart) evaluó predictores de desenlaces en embarazadas con enfermedad cardíaca congénita y encontró tabaquismo, disfunción del ventrículo derecho e insuficiencia pulmonar severa, como predictores de desenlaces adversos ${ }^{21}$. En este estudio el tabaquismo se relacionó con el desarrollo de hemorragia postparto $(\mathrm{p}<0.001)$ y arritmia sintomática sostenida que requiere tratamiento ( $p$ 0.017), mientras que el uso de warfarina durante la gestación se relacionó con muerte neonatal ( $p$ 0.049) y el desarrollo de arritmia sintomática sostenida que requirió tratamiento ( $\mathrm{p} 0.049)$.

Los factores de riesgo para complicaciones perinatales que han sido estudiados son pobre clase funcional NYHA, obstrucción del corazón izquierdo, edad menor a 20 o mayor a 35 años, embarazo múltiple, tabaquismo y anticoagulación durante la gestación ${ }^{2}$.

En un estudio de embarazadas con enfermedad cardíaca congénita, los desenlaces obstétricos adversos, como ruptura prematura de membranas (RPM), hemorragia postparto y parto pretérmino, ocurrieron en el $32 \%$ de las pacientes ${ }^{14}$. En este estudio los desenlaces adversos obstétricos fueron hemorragia postparto (2.4\%), RPM (7.3\%) y desarrollo de THAE (29.3\%).

En cuanto a los hallazgos ecocardiográficos, se ha intentado encontrar asociación entre pobre función sistólica del ventrículo izquierdo, hipertensión pulmonar, insuficiencia tricuspídea moderada/severa, disfunción del ventrículo derecho y volumen indexado de la aurícula izquierda, con desenlaces adversos maternos y neonatales. En un estudio realizado por nuestro grupo de investigación se encontró que las pacientes con presión sistólica pulmonar $\geq 50 \mathrm{~mm}$ Hg tuvieron mayor frecuencia de eventos maternos cardíacos primarios y secundarios. Además, al evaluar el volumen indexado de la aurícula izquierda se observó que pacientes con volumen auricular mayor a $34 \mathrm{ml} / \mathrm{m}^{2}$ tenían mayor frecuencia de eventos neonatales, hallazgo que no había sido reportado previamente en la literatura ${ }^{12}$. 
El pronóstico de pacientes embarazadas con hipertensión pulmonar debida a enfermedad del corazón izquierdo es más favorable comparado con la presencia de hipertensión arterial pulmonar del grupo 1. En hipertensión arterial pulmonar idiopática la mortalidad se ubica entre el 10 al $17 \%$ y en aquella secundaria a síndrome de Eisenmenger entre el 23 al $28 \% 5,27,28$. El pronóstico de embarazadas con hipertensión pulmonar puede ser más favorable cuando esta es leve (PSP $<50 \mathrm{~mm} \mathrm{Hg}$ ) o cuando se asocia a clase funcional I-II de la $\mathrm{NYHA}^{29,30}$.

En este estudio las pacientes con FEVI mayor a $55 \%$ tuvieron, en su mayoría, recién nacidos vivos, la presencia de insuficiencia tricuspídea moderada/severa se asoció con prematuridad y el desarrollo de arritmia sintomática sostenida requirió tratamiento; las gestantes con PSP $\geq 50 \mathrm{~mm} \mathrm{Hg}$ tuvieron mayor número de neonatos con distrés respiratorio y el volumen indexado de la aurícula izquierda (crecimiento moderado/severo) se asoció con el desarrollo de arritmia sintomática sostenida que requirió tratamiento y necesidad de terapia invasiva antes de seis meses postparto.

La falla cardiaca derecha se relacionó con muerte materna y prematuridad mientras que la de falla cardiaca izquierda se asoció con muerte neonatal.

Aproximadamente el $30 \%$ de las mujeres con condiciones cardiovasculares crónicas tomaba alguna medicación; entre ellas las más frecuentes fueron los betabloqueadores, los inhibidores de la enzima convertidora de la angiotensina (IECA), los diuréticos y los agentes antiplaquetarios ${ }^{21}$. En este estudio la medicación cardiovascular más utilizada durante el embarazo fueron las heparinas (43.9\%), los betabloqueadores $(34.1 \%)$, los diuréticos $(31.7 \%)$, los calcioantagonistas $(12.2 \%)$, la warfarina $(9,8 \%)$ y el ASA $(7.3 \%)$. Esto refleja la complejidad de la enfermedad cardiovascular durante el embarazo y los cuidados que se deben tener para evitar medicación con alto potencial teratogénico y riesgo de eventos adversos neonatales, como los IECA y bloqueadores del receptor de angiotensina II.

\section{Limitaciones del estudio}

Se trata de un estudio observacional descriptivo en el que no pueden evaluarse asociaciones causales. En primer lugar, es un estudio retrospectivo con revisión de historias clínicas en las cuales pudieron haber existido omisiones en el registro de los datos por parte del personal asistencial. En segundo lugar, el tamaño pequeño de la muestra no permite que las asociaciones encontradas entre las variables objeto del estudio tengan el suficiente poder estadístico. Por último, el diagnóstico de hipertensión pulmonar se realizó con base en los hallazgos ecocardiográficos, así que pudo haberse sobrediagnosticado e incluido un mayor número de pacientes en el grupo de riesgo IV de la OMS. Se requieren estudios con mayor número de pacientes, en múltiples centros y con protocolos de manejo estandarizados que permitan evaluar de manera más precisa los desenlaces.

\section{Conclusiones}

Es frecuente la asociación de enfermedad cardiovascular y embarazo; por este motivo, es importante identificar este tipo de pacientes para poder brindar desde etapas muy tempranas, idealmente en la etapa preconcepcional, adecuada asesoría y acompañamiento a cargo de un equipo multidisciplinario. Se requieren más estudios en el contexto colombiano que identifiquen de manera oportuna los desenlaces maternos y fetales. Se destaca el papel de la ecocardiografía como herramienta clave para la evaluación y estratificación no invasiva del riesgo de las pacientes embarazadas.

\section{Financiación}

Universidad CES y Hospital General de Medellín.

\section{Conflicto de intereses}

Ninguno.

\section{Agradecimientos}

A la Universidad CES y al Hospital General de Medellín.

\section{Bibliografía}

1. Hall ME, George EM, Granger JP. El corazón durante el embarazo. Rev Esp Cardiol. 2011;64:1045-50.

2. Elkayam U, Goland S, Pieper PG, Silversides CK. High-risk cardiac disease in pregnancy. J Am Coll Cardiol. 2016;68:396-410.

3. Endorsed by the European Society of Gynecology (ESG), the Association for European Paediatric Cardiology (AEPC), and the German Society for Gender Medicine (DGesGM), Authors/Task Force Members, Regitz-Zagrosek V, Blomstrom Lundqvist C, Borghi C, Cifkova R, et al. ESC Guidelines on the management of cardiovascular diseases during pregnancy: The Task Force on the Management of Cardiovascular Diseases during Pregnancy of the European Society of Cardiology (ESC). Eur Heart J. 2011;32(24):3147-97.

4. Roos-Hesselink JW, Ruys TPE, Stein JI, Thilen U, Webb GD, Niwa $\mathrm{K}$, et al. Outcome of pregnancy in patients with structural or ischaemic heart disease: results of a registry of the European Society of Cardiology. Eur Heart J. 2013;34:657-65.

5. Elkayam U, Goland S, Pieper PG, Silversides CK. High-risk cardiac disease in pregnancy. J Am Coll Cardiol. 2016;68:502-16.

6. Regitz-Zagrosek V, Gohlke-Bärwolf C, lung B, Pieper PG. Management of Cardiovascular Diseases During Pregnancy. Curr Probl Cardiol. 2014;39(4-5):85-151.

7. Hebson C, Saraf A, Book WM. Risk assessment and management of the mother with cardiovascular disease. Clin Perinatol. 2016;43:1-22.

8. Lewey J, Haythe J. Cardiomyopathy in pregnancy. Semin Perinatol. 2014;38:309-17.

9. Canobbio MM, Warnes CA, Aboulhosn J, Connolly HM, Khanna $\mathrm{A}$, Koos BJ, et al. Management of pregnancy in patients with complex congenital heart disease: a scientific statement for healthcare professionals from the American Heart Association. Circulation. 2017;135:e50-87.

10. Hameed AB, Sklansky MS. Pregnancy: maternal and fetal heart disease. Curr Probl Cardiol. 2007;32:419-94.

11. Hu H, Pasca I. Management of complex cardiac issues in the pregnant patient. Crit Care Clin. 2016;32:97-107.

12. Múnera AG, Manrique R, Orrego J, Mesa C, Betancur C, Gómez M. Valor pronóstico de los estudios ecocardiográficos en el diagnóstico y seguimiento de patología cardíaca durante el embarazo. Rev Fed Arg Cardiol. 2012;41:114-20. 
13. Ouyang DW, Khairy P, Fernandes SM, Landzberg MJ, Economy KE. Obstetric outcomes in pregnant women with congenital heart disease. Int J Cardiol. 2010;144:195-9.

14. Balci A, Sollie-Szarynska KM, van der Bijl AGL, Ruys TPE, Mulder BJM, Roos-Hesselink JW, et al. Prospective validation and assessment of cardiovascular and offspring risk models for pregnant women with congenital heart disease. Heart. 2014;100:1373-81.

15. Gelson E, Curry R, Gatzoulis MA, Swan L, Lupton M, Steer P, et al. Effect of Maternal Heart Disease on Fetal Growth: Obstet Gynecol. 2011;117:886-91.

16. Thorne S. Risks of contraception and pregnancy in heart disease. Heart. 2006;92:1520-5.

17. Knight M, Kenyon S, Brocklehurst P, Neilson J, Shakespeare J, Kurinczuk JJE. Saving Lives, Improving Mothers' Care Lessons learned to inform future maternity care from the UK and Ireland Confidential Enquiries into Maternal Deaths and Morbidity 20092012. Oxford national perinatal epidemiology unit, University of Oxford;. 2014

18. Siu SC, Sermer M, Colman JM, Alvarez AN, Mercier L-A, Morton BC, et al. Prospective Multicenter Study of Pregnancy Outcomes in Women With Heart Disease. Circulation. 2001;104:515-21.

19. Siu SC. Adverse Neonatal and Cardiac Outcomes Are More Common in Pregnant Women With Cardiac Disease. Circulation. 2002;105:2179-84.

20. Drenthen W, Boersma E, Balci A, Moons P, Roos-Hesselink JW, Mulder BJM, et al. Predictors of pregnancy complications in women with congenital heart disease. Eur Heart J. 2010;31:2124-32.

21. Khairy P. Pregnancy outcomes in women with congenital heart disease. Circulation. 2006;113:517-24.

22. Sillesen M, Hjortdal V, Vejlstrup N, Sørensen K. Pregnancy with prosthetic heart valves - 30 years' nationwide experience in Denmark. Eur J Cardiothorac Surg [Internet]. 2011 Jan 28 [Acceso 14 May2018];
Disponible en: https://academic.oup.com/ejcts/articlelookup/doi/10.1016/j.ejcts.2010.12.011.

23. Goland S, Elkayam U. Anticoagulation in Pregnancy. Cardiol Clin. 2012;30:395-405.

24. van Hagen IM, Roos-Hesselink JW, Ruys TPE, Merz WM, Goland S, Gabriel H, et al. Pregnancy in women with a mechanical heart ValveCLINICAL PERSPECTIVE: Data of the European Society of Cardiology Registry of Pregnancy and Cardiac Disease (ROPAC). Circulation. 2015;132:132-42.

25. Drenthen W, Pieper PG, Roos-Hesselink JW, van Lottum WA, Voors AA, Mulder BJM, et al. Outcome of pregnancy in women with congenital heart disease. J Am Coll Cardiol. 2007; 49:11-2303.

26. Sm SCS, Sermer M, Harrison DA, Grigoriadis E, Liu G, Sorensen S, et al. Risk and predictors for pregnancy-related complications in women with heart disease. Circulation. 1997;96:94-2789.

27. Galiè N, Humbert M, Vachiery J-L, Gibbs S, Lang I, Torbicki A, et al. 2015 ESC/ERS Guidelines for the diagnosis and treatment of pulmonary hypertension: The Joint Task Force for the Diagnosis and Treatment of Pulmonary Hypertension of the European Society of Cardiology (ESC) and the European Respiratory Society (ERS)Endorsed by: Association for European Paediatric and Congenital Cardiology (AEPC) International Society for Heart and Lung Transplantation (ISHLT). Eur Heart J. 2016;37:67-119.

28. Pieper PG, Lameijer H, Hoendermis ES. Pregnancy and pulmonary hypertension. Best Pract Res Clin Obstet Gynaecol. 2014;28:579-91.

29. Katsuragi S, Yamanaka K, Neki R, Kamiya C, Sasaki Y, Osato K, et al. Maternal outcome in pregnancy complicated with pulmonary arterial hypertension. Circ J. 2012;;76:54-2249.

30. Subbaiah M, Kumar S, Roy KK, Sharma JB, Singh N. Pregnancy outcome in women with pulmonary arterial hypertension: single-center experience from India. Arch Gynecol Obstet. 2013;288:305-9. 